\title{
Teleparallel Gravity: Foundations and Observational Constraints-Editorial
}

\author{
Sebastian Bahamonde ${ }^{1, *}$ and Jackson Levi Said ${ }^{2,3}$ (D) \\ 1 Laboratory of Theoretical Physics, Institute of Physics, University of Tartu, W. Ostwaldi 1, 50411 Tartu, Estonia \\ 2 Institute of Space Sciences and Astronomy, University of Malta, MSD 2080 Msida, Malta; \\ jackson.said@um.edu.mt \\ 3 Department of Physics, University of Malta, MSD 2080 Msida, Malta \\ * Correspondence: sbahamonde@ut.ee
}

Citation: Bahamonde, S.; Said, J.L. Teleparallel Gravity: Foundations and Observational Constraints-Editorial. Universe 2021, 7, 269. https:// doi.org/10.3390/universe7080269

Received: 23 July 2021

Accepted: 23 July 2021

Published: 27 July 2021

Publisher's Note: MDPI stays neutral with regard to jurisdictional claims in published maps and institutional affiliations.

Copyright: (c) 2021 by the authors. Licensee MDPI, Basel, Switzerland. This article is an open access article distributed under the terms and conditions of the Creative Commons Attribution (CC BY) license (https:/ / creativecommons.org/licenses/by/ $4.0 /)$.
Einstein's formulation of general relativity as a theory based on the geometry of curvature was a necessity due to Riemannian geometry being the only fully developed framework at the time [1]. However, less than a decade after this time, Einstein was able to consider an important alternative geometry, where torsion would substitute curvature in the geometric deformation produced by gravity [2]. At this time and over the following decades the work of Roland Weitzenböck, Élie Cartan, Luther P. Eisenhart, Herman Müntz, Jakob Grommer and Cornelius Lanczos was pivotal to this quest. Einstein's original motivation was to formulate a theory in which electromagnetism would form part of a fuller theory in which gravity would simply be part of the unification. However, it was not long until it was found out that the extra degrees of freedom in the new torsional theory of gravity were not reconcilable with electromagnetism since they were already associated with the local Lorentz group. This prompted Einstein and some others to largely abandon the theory.

After a number of revivals, the foundations of teleparallel gravity has been significantly improve both in formulation and understanding, and is now able to produce a competitive teleparallel equivalent to general relativity (TEGR) [3]. This theory is guaranteed to produce all the classical results of general relativity. However, it may have a more tame quantum limit opening the possibility of a more behaved quantum theory of gravity. Another facet of this formulation of gravity is its numerical efficiency since it is algebraically different [4]. This comes into play when performing simulations within numerical relativity which can take months to run in certain circumstances such as template production for black hole observatories. TEGR is very interesting theoretically as well because it revives gravity as a force again bringing it closer to our understanding of electromagnetism. Another crucial aspect of TEGR is that it organically contains a Gibbons-Hawking-York boundary term in its action giving it a well-defined Hamiltonian expression giving it some advantage over general relativity in this respect.

In recent decades there have been numerous proposals for modifying TEGR, similar to general relativity there are many directions in which to consider different formulations of gravity. While quantum aspects of the theory have not been well developed yet, there has been a strong impetus to study possible generalizations using the scalars associated with the theory such as its TEGR Lagrangian which is called torsion scalar. This theory also produces novel scalars such as the boundary term which represents the difference between the TEGR and Einstein-Hilbert action Lagrangian terms. On the other hand, teleparallel gravity also produces scalars that appear in all gravitational frameworks such as the GaussBonnet term among others [5]. Teleparallel gravity has been probed for its extensions into the field of scalar-tensor theories where a scalar field is coupled with some of the contributions to the gravitational sector. Primarily this has occurred in the cosmological section due to this being a key area for modified gravity to succeed in. One such theory in this regime is the recently proposed teleparallel analogue of Horndeski theory which is 
a teleparallel version of Horndeski's famous theory of gravity which has almost become synonymous with modified gravity in regular curvature-based theories of gravity [6].

Teleparallel gravity [7] and its modifications continues to produce intriguing and novel results, one area that has lacked significant progress in the literature is that of astrophysics which is a poorly studied arm within the theory. Much work is still needed to understand the nature and behaviour of black holes in teleparallel gravity. We also do not yet fully understand how astrophysical dark matter may or may not play a role in these modified teleparallel theories of gravity.

This special issue of Universe is taking place at a timely moment when teleparallel gravity appears to be entering a critical point in its development with several foundational questions coming to the fore and, on the data analysis side, serious tools being developed to better probe its observational impacts in precision cosmology [8]. To this end, we have ten interesting articles that touch on the various topics across teleparallel gravity. On the quantum gravity side, the issue has an article that proposes an approach in which special relativity is slightly altered to improve the quantum gravity behaviour of TEGR [9], while another explored the possibility of using recent arguments of entropy to produce a quantization of gravity in various settings [10]. Staying on the foundations side of things, in another article the range of possible variational principles from which teleparallel theories can be dynamically formulated is investigated [11], which is complimented by another article which explored the fate of symmetries in the various formulations of teleparallel gravity [12].

In the astrophysics regime, the problem of black holes in teleparallel gravity is probed through the weak field in $f(T)$ gravity with interesting solutions and predictions being found [13]. Perturbations in the setting of spherical symmetry is again used in another article which studies more general approaches to producing such solutions [14]. Finally, the quasinormal modes of a Schwarzschild black hole are examined in another article on the topic [15]. On the cosmology side of things, the issue has three articles touching on several critical points in the development of any theory of gravity, namely the behaviour of teleparallel gravity in light of inflationary processes [16], together with the possible quantization through a minisuperspce approach [17], and the production of particles and the stability of this process in the matter coupling scenario of the theory [18].

Funding: This research received no external funding.

Conflicts of Interest: The authors declare no conflict of interest.

\section{References}

1. Misner, C.W.; Thorne, K.S.; Wheeler, J.A. Gravitation; Freeman: England, UK, 1973.

2. Goenner, H.F.M. On the History of Unified Field Theories. Part II. (ca. 1930-ca. 1965). Living Rev. Relat. 2014, 17, 5. [CrossRef] [PubMed]

3. Unzicker, A.; Case, T. Translation of Einstein's Attempt of a Unified Field Theory with Teleparallelism. arXiv 2005, arXiv:physics $/ 0503046$.

4. Aldrovandi, R.; Pereira, J.G. Teleparallel Gravity: An Introduction; Springer: Berlin/Heidelberg, Germany, 2013.

5. Kofinas, G.; Saridakis, E.N. Teleparallel equivalent of Gauss-Bonnet gravity and its modifications. Phys. Rev. D 2014, 90, 084044. [CrossRef]

6. Cai, Y.-F.; Capozziello, S.; De Laurentis, M.; Saridakis, E.N. $f(T)$ teleparallel gravity and cosmology. Rep. Prog. Phys. 2016, 79, 106901. [CrossRef] [PubMed]

7. Krššák, M.; van den Hoogen, R.J.; Pereira, J.G.; Böhmer, C.G.; Coley, A.A. Teleparallel theories of gravity: Illuminating a fully invariant approach. Class. Quantum Gravity 2019, 36, 183001. [CrossRef]

8. Bahamonde, S.; Dialektopoulos, K.F.; Escamilla-Rivera, C.; Farrugia, G.; Gakis, V.; Hendry, M.; Hohmann, M.; Said, J.L.; Mifsud, J.; Di Valentino, E. Teleparallel Gravity: From Theory to Cosmology. arXiv 2021, arXiv:2106.13793.

9. Pereira, J.G.; López, D.F. An Improved Framework for Quantum Gravity. Universe 2020, 6, 243. [CrossRef]

10. Bajardi, F.; Bascone, F.; Capozziello, S. Renormalizability of Alternative Theories of Gravity: Differences between Power Counting and Entropy Argument. Universe 2021, 7, 148. [CrossRef]

11. Hohmann, M. Variational Principles in Teleparallel Gravity Theories. Universe 2021, 7, 114. [CrossRef]

12. Beltrán Jiménez, J.; Koivisto, T.S. Accidental Gauge Symmetries of Minkowski Spacetime in Teleparallel Theories. Universe 2021, 7, 143. [CrossRef] 
13. Pfeifer, C.; Schuster, S. Static spherically symmetric black holes in weak $\mathrm{f}(\mathrm{t})$-gravity. Universe 2021, 7, 153. [CrossRef]

14. Golovnev, A.; Guzmán, M.-J. Approaches to Spherically Symmetric Solutions in f(T) Gravity. Universe 2021, 7, 121. [CrossRef]

15. Maluf, J.W.; Ulhoa, S.; Carneiro, F.L.; Castello-Branco, K.H.C. Perturbations of the Gravitational Energy in the TEGR: Quasinormal Modes of the Schwarzschild Black Hole. Universe 2021, 7, 100. [CrossRef]

16. Järv, L.; Lember, J. Global Portraits of Nonminimal Teleparallel Inflation. Universe 2021, 7, 179. [CrossRef]

17. Paliathanasis, A. Minisuperspace Quantization of $\mathrm{f}(\mathrm{T}, \mathrm{B})$ Cosmology. Universe 2021, 7, 150. [CrossRef]

18. Harko, T.; Lobo, F.S.N.; Saridakis, E.N. Gravitationally Induced Particle Production through a Nonminimal Torsion-Matter Coupling. Universe 2021, 7, 227. [CrossRef] 